\title{
Localized Charge Transfer Reactions near the Pt-YSZ Interfaces using Kelvin Probe Microscopy
}

\author{
Wonyoung Lee ${ }^{1,2.7}$ and Fritz B. Prinz $z^{2,3}$ \\ 1 School of Mechanical Engineering, Sungkyungkwan University, 2066 Seobu-ro, Jangan-gu Suwon, Kyunggi-do 440-746, South Korea \\ 2 Department of Mechanical Engineering. Stanford University, 440 Escondido Mall, Stanford, CA 94305, USA \\ 3 Department of Materials and Science Engineering, Stanford University, 440 Escondido Mall, Stanford, CA 94305, USA \\ \# Corresponding Author / E-mail: leewy@skku.edu, TEL: +82-31-299-4848, FAX: +82-31-290-5889
}

KEYWORDS: Kelvin probe microscopy, Solid oxide fuel cell, Charge transfer reaction, Interface

Localized charge transfer reactions induced by the external bias were observed near the electrode-electrolyte interfaces by Kevin probe microscopy. Spatially resolved potential measurements revealed the localized charge accumulation and rearrangement driven by the external bias, which were ascribed to oxidation and reduction of the oxide surface. This in situ measurement of charge distribution with spatial information under controlled environments can be particularly useful in investigating the charge transfer reactions on the surfaces of functional materials and enhance our understanding of many electronic and electrochemical devices.

Manuscript received: April 26, 2014 / Revised: May 17, 2014 / Accepted: May 19, 2014

\section{Introduction}

Ionic charge transfer reactions at the surfaces of functional materials are of key importance for many electrochemical devices to improve the device performance, including solid oxide fuel cells, ${ }^{1}$ lithium ion batteries, ${ }^{2}$ and gas sensors. ${ }^{3}$ Particularly, mechanisms of oxidation and reduction at the surfaces of oxide ion conductors have been extensively investigated since the rate of charge transfer reactions is a major factor for the overall performance of electrochemical devices. ${ }^{4}$ Performance enhancement of these applications requires localized probing of complex ionic phenomena with high spatial resolution, uncovering the underlying physics and correlating to a macroscopic device or material functionality.

A variety of characterization methods has been employed to investigate charge transfer reactions, including photoelectron emission microscopy (PEEM), scanning photoelectron microscopy (SPEM), ${ }^{5,6}$ Kelvin probe, ${ }^{7}$ scanning transmission electron spectroscopy (STEM), ${ }^{8}$ and secondary ion mass spectroscopy (SIMS). ${ }^{9}{ }^{10}$ However, in situ studies allowing direct manipulation and observation of a local rearrangement of charged species are scarce. Atomic force microscopy (AFM) has become one of the primary tools to interrogate the local properties of a variety of materials at the nanoscale. Recent progress of AFM to probe local ionic and electronic transport, dielectric, optical, ferroelectric, and magnetic properties has made the technique a more powerful platform to investigate the complex charge transfer reactions of functional surfaces and interfaces at the nanoscale. ${ }^{11,12}$ The conductive tip can be used as an electrode to detect the potential distribution on the oxide surface. Moreover, the precise positioning capability of AFM allows in situ creation and observation of charge transfer reactions under controlled environments. ${ }^{11-13}$

In this study, we investigated the localized charge transfer reactions on the oxide surface using conductive AFM. We examined the interfaces between platinum ( $\mathrm{Pt}$ ) and yttria-stabilized zirconia (YSZ) as a model system of the electrode-electrolyte interfaces in solid oxide fuel cells. ${ }^{1,4}$ Kelvin probe microscopy (KPM) was employed to observe the local charge distribution on the surface with custom-built setups to decouple the geometric convolution. ${ }^{14,15}$ We showed correlation between the measured potential distribution near the Pt-YSZ interfaces under various external bias conditions and charge transfer reactions.

\section{Experimental Details}

We employed a conventional AFM system (JSPM-5200, JEOL) with modification for surface potential measurements as shown in Fig. 1(a). Green solid lines represent the circuitry for modified KPM, and experimental details were described in our earlier publication. ${ }^{15}$ Blue dashed lines represent the circuitry to apply external bias to the $\mathrm{Pt}$ 
(a)

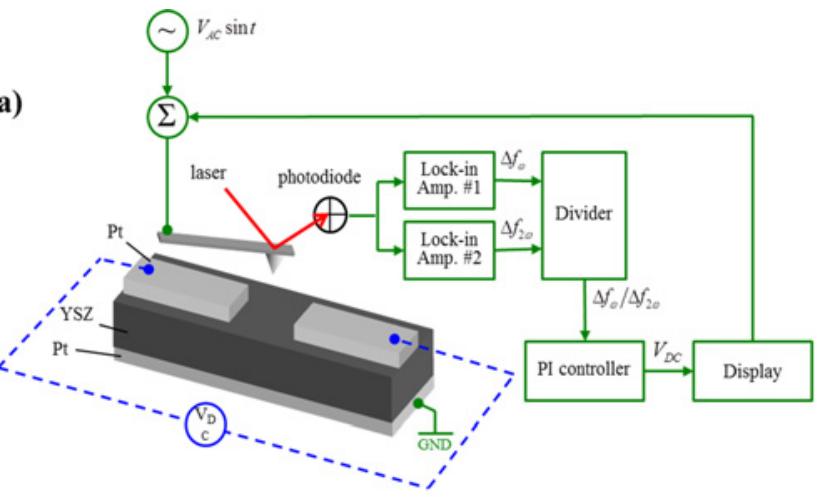

(b)

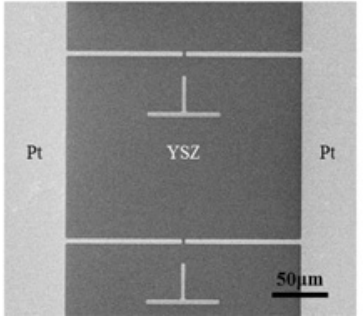

(c)

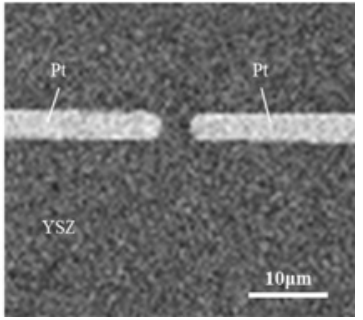

Fig. 1 (a) Schematic for surface potential measurements Green and blue lines represent the schematic for modified KPM setups, and the external DC bias to Pt patterns, respectively. (b) SEM image of Pt patterns on the YSZ films (low magnification), and (c) SEM image of Pt patterns on the YSZ films (high magnification)

electrodes. All circuitries shared the common ground, which was used to apply $0 \mathrm{~V}$ to the electrode. Measurements were performed using frequency modulation (FM) non-contact modes to avoid the contact between the tip and the sample surface during bias application. ${ }^{15,16}$ Topography and surface potential were simultaneously measured in vacuum $\left(1 \times 10^{-6}\right.$ Torr $)$ at room temperature. Pt coated conductive probes with a spring constant of $\sim 40 \mathrm{Nm}^{-1}$ and a resonance frequency of $\sim 150 \mathrm{kHz}$ (Nanosensors) were used for FM mode imaging.

We deposited a $200 \mathrm{~nm}$ thick Pt layer on a silicon nitride film as a reference/counter electrode by DC sputtering. YSZ films were subsequently deposited on the Pt layer at $200^{\circ} \mathrm{C}$ by radio-frequency (RF) sputtering using the $\mathrm{Y}_{0.16} \mathrm{Zr}_{0.84} \mathrm{O}_{1.92} \mathrm{RF}$ sputtering target (Kurt Lesker Co.). ${ }^{17}$ Conventional photolithography, DC sputtering, and liftoff processes were used to fabricate $20 \mathrm{~nm}$ thick Pt patterns on the YSZ film. The width of each Pt finger and the gap between Pt fingers were $\sim 5 \mu \mathrm{m}$ as shown in Figs. 1(b-c). All samples were cleaned before measurements in the $\mathrm{AFM}$ chamber at $120^{\circ} \mathrm{C}$ for $1 \mathrm{hr}$ to eliminate possible contamination on the surface.

\section{Results and Discussion}

To assess the charge transfer reactions near the electrode-electrolyte interfaces, we measured the potential distribution using modified KPM. Applying external DC bias to Pt electrodes on the YSZ surface enabled the investigation of the changes in the potential distribution under polarized conditions. In particular, oxidation and reduction of YSZ films with the positive and negative potential, respectively, could be examined as a function of the applied external bias with spatial information.
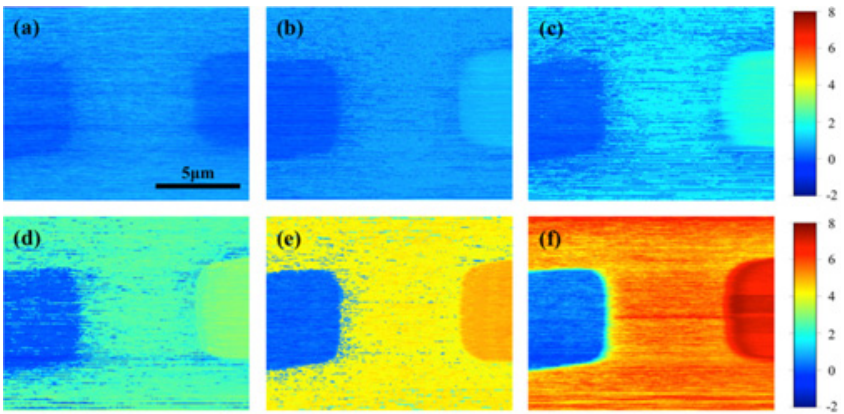

Fig. 2 Measured potential maps near the Pt-YSZ interfaces with various bias conditions. A left electrode was set to $0 \mathrm{~V}$ for all maps. A right electrode was set to (a) $0 \mathrm{~V}$, (b) $1 \mathrm{~V}$, (c) $2 \mathrm{~V}$, (d) $3 \mathrm{~V}$, (e) $5 \mathrm{~V}$, and (f) $6.8 \mathrm{~V}$

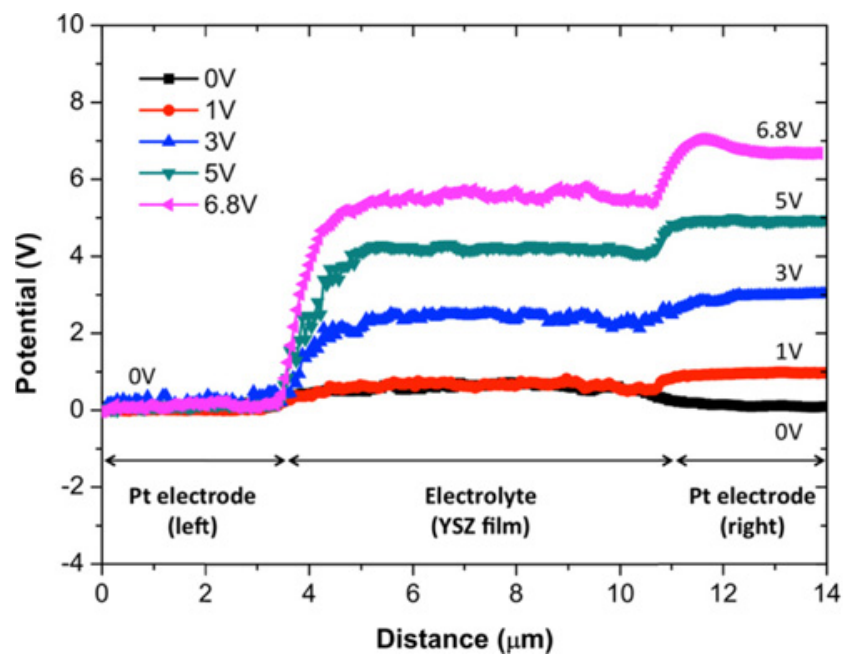

Fig. 3 Potential profiles between the Pt electrodes on the YSZ surface

Fig. 2 shows the measured potential maps with various DC bias conditions. The left electrode was set to $0 \mathrm{~V}$ for all measurements, while the right electrode was varied from $0 \mathrm{~V}$ to $6.8 \mathrm{~V}$. The series of potential maps showed the evolution of potential variation near the PtYSZ interfaces with the external DC biases. The modified KPM setups were employed to suppress the geometrical convolution, which is otherwise difficult to avoid due to the finite size of the tip radius. ${ }^{12,15,18}$ Fig. 3 shows the potential profiles between two Pt electrodes on the YSZ surface. The measured potential values were well matched with the applied DC bias to the electrodes, validating the accuracy of the measurement scheme.

In order to investigate the effects of the external bias on the potential distribution near the Pt-YSZ interfaces, we obtained the potential maps before and after the external bias application to the $\mathrm{Pt}$ electrodes. Fig. 4(a) shows the initial potential distribution with $0 \mathrm{~V}$ to both electrodes. The difference in potential between the Pt electrodes and the YSZ films originates from the work function difference between Pt and YSZ. ${ }^{6,19}$ While keeping $0 \mathrm{~V}$ to the left electrode, we applied $6.8 \mathrm{~V}$ to the right electrode in the vacuum chamber $\left(1 \times 10^{-6}\right.$ Torr) at room temperature for $2 \mathrm{hrs}$. Then, we applied $0 \mathrm{~V}$ to both electrodes to see the changes in potential distribution near the Pt-YSZ interfaces induced by the external bias application. Fig. 4(b) presents the resultant potential distribution showing substantial changes in the potential distribution near the Pt-YSZ interfaces. The negative and 
(a)

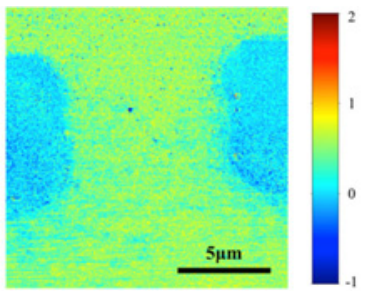

(b)
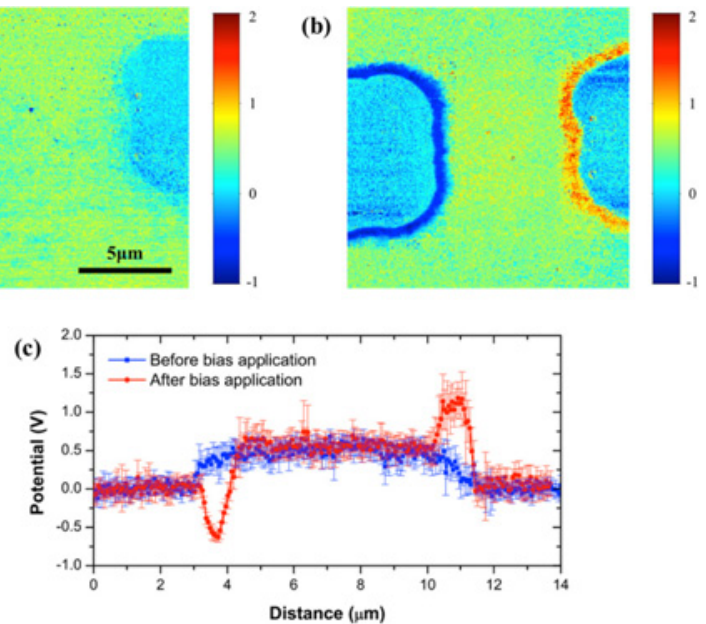

Fig. 4 Potential maps near the Pt-YSZ interfaces with $0 \mathrm{~V}$ for both electrodes (a) before $6.8 \mathrm{~V}$ application to the right electrode, and (b) after $6.8 \mathrm{~V}$ application to the right electrode. (c) The potential profile between the Pt electrodes

positive potential features were observed around the left and right electrode, respectively. These potential features remained unchanged for more than 2 hrs.

Observed changes in the potential distribution near the Pt-YSZ interfaces can be ascribed to charge accumulation by applying the external bias to the Pt electrodes. The negative potential features can be attributed to electron injection into the YSZ surface reported as electrifications. ${ }^{20,21}$ Injected electrons from the Pt electrode to the YSZ surface could be trapped in defects on the surface, such as oxide ion vacancies, resulting in the reduction of the YSZ surface. ${ }^{6,22}$ Trapped electrons may dissipate over time via incorporation with charged species on the surface, particularly the surface water layer. However, combination of the heat treatment at $120^{\circ} \mathrm{C}$ for $1 \mathrm{hr}$ and the subsequent measurements in vacuum $\left(1 \times 10^{-6}\right.$ Torr $)$ eliminated, or at least significantly reduced, the surface water layer, and which caused the retarded charge dissipation rate on the surface. ${ }^{13}$ In contrast, the positive potential features can be attributed to the formation and accumulation of oxide ion vacancies. The positive bias applied to the Pt electrode can take electrons from oxide ions on the YSZ surface, resulting in the oxidation of the YSZ surface. Losing electrons to the Pt electrode allowed $\mathrm{O}_{2}$ molecules to depart from the crystal surface and oxide ion vacancies to be formed near the Pt-YSZ interfaces. The low diffusivity of oxide ion vacancies at given temperature and oxygen partial pressure can account for the retarded charge dissipation rate. ${ }^{23,24}$

Lastly, we investigated the reversibility of potential distribution by reversing the external bias. Figs. 5(a-c) show the potential distribution during the bias application of $0 \mathrm{~V}$ to the left electrode and $6.8 \mathrm{~V}$ to the right electrode (Fig. 5(a)), after resuming $0 \mathrm{~V}$ to both electrodes (Fig. 5(b)), and the potential profile (Fig. 5(c)). Consistent with the previous observation, the reduction and oxidation of the YSZ surfaces occurred as indicated by the negative and positive potential features near the left and right electrodes, respectively. Then, we applied $6.8 \mathrm{~V}$ to the left electrode and $0 \mathrm{~V}$ to the right electrode, reversing bias, and resumed to $0 \mathrm{~V}$ to both electrodes. Figs. 5(d-e) show the potential distribution during the bias application (Fig. 5(d)), after resuming $0 \mathrm{~V}$ to both electrodes (Fig. 5(e)), (a)

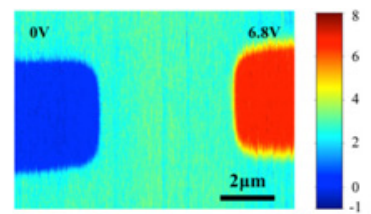

(b)

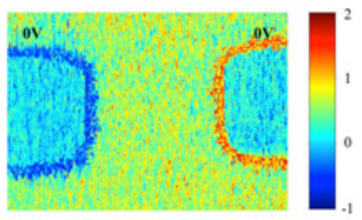

(c)

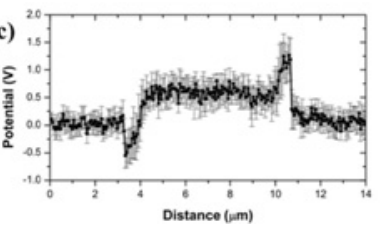

(d)

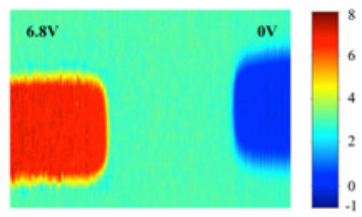

(e)

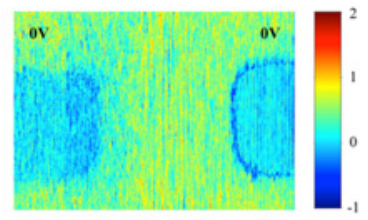

(f)

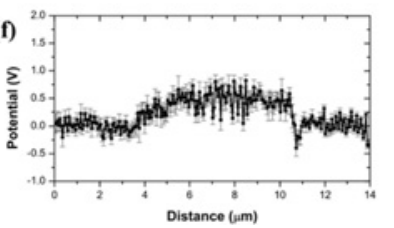

Fig. 5 A series of potential maps near the Pt-YSZ interfaces (a) during bias application of $0 \mathrm{~V}$ to the left electrode and $6.8 \mathrm{~V}$ to the right electrode, (b) after resuming $0 \mathrm{~V}$ to both electrodes, (c) potential profile between the Pt electrodes, (d) during bias application of $6.8 \mathrm{~V}$ to the left electrode and $0 \mathrm{~V}$ to the right electrode, (e) after resuming $0 \mathrm{~V}$ to both electrodes, and (f) potential profile between the $\mathrm{Pt}$ electrodes

and the potential profile (Fig. 5(f)). Interestingly, reversing the bias to the electrode converted the polarity of the potential features. The positive potential features around the right electrode became negative, indicating the reduction of the YSZ surface. We believe that injection of electrons into the region with accumulated oxide ion vacancies converted the contrast of the potential features, which corresponded to the electron accumulation. The fact that the negative potential features around the left electrode disappeared can be explained by extraction of electrons from the region with accumulated electrons and creation of oxide ion vacancies, converting the contrast of the potential features. However, oxidation of the reduced YSZ surface may be more demanding than reduction of the oxidized YSZ surface, and therefore, the contrast of the potential features were converted partially from negative to positive.

\section{Conclusions}

We demonstrated the spatially resolved potential measurements near the Pt-YSZ interfaces to investigate the charge transfer reactions under various bias conditions. Applying the external bias induced the accumulation of charged species near the Pt-YSZ interfaces, and the polarity of the external bias determined the types of accumulated species with opposite charges, as observed by AFM-based potential measurements. Negative potential features corresponded to oxidation of the YSZ surface due to electron injection into the YSZ surfaces. Positive potential features, in contrast, corresponded to the reduction of the YSZ surface due to creation of oxide ion vacancies. Reversing the bias polarity converted the contrast of measured potential features, indicating that the reverse reaction occurred. This observation confirmed that the external bias caused and rearranged the charge configuration and distribution near the Pt-YSZ interfaces. This 
technique can be particularly useful for investigating the charge transfer reactions and charge distribution near the surface reaction sites since it can provide in situ observation of localized charge distribution with spatial information. When combined with more control of external environments, including temperature and reactant gas, this can be even more powerful to explore the localized charge transfer reactions under actual operating conditions.

\section{ACKNOWLEDGEMENT}

This research was supported by Basic Science Research Program through the National Research Foundation of Korea (NRF) funded by the Ministry of Science, ICT \& Future Planning (Grant No. NRF2013R1A1A1059845).

\section{REFERENCES}

1. O'Hayre, R. P., Cha, S. W., Colella, W., and Prinz, F. B., "Fuel Cell Fundamentals," Wiley, $1^{\text {st }}$ Ed., 2006.

2. Nazri, G. A. and Pistoia, G., "Lithium Batteries: Science and Technology," Springer Science \& Business, $1^{\text {st }}$ Ed., 2008.

3. Bakker, E. and Qin, Y., "Electrochemical Sensors," Analytical Chemistry, Vol. 78, No. 12, pp. 3965-3984, 2006.

4. Adler, S. B., "Factors Governing Oxygen Reduction in Solid Oxide Fuel Cell Cathodes," Chemical Reviews, Vol. 104, No. 10, pp. 4791-4844, 2004.

5. Janek, J., Luerssen, B., Mutoro, E., Fischer, H., and Günther, S., "In Situ Imaging of Electrode Processes on Solid Electrolytes by Photoelectron Microscopy and Microspectroscopy-The Role of the Three-phase Boundary," Topics in Catalysis, Vol. 44, No. 3, pp. 399407, 2007.

6. Luerßen, B., Janek, J., Günther, S., Kiskinova, M., and Imbihl, R., "Microspectroscopy at a Moving Reduction Front in Zirconia Solid Electrolyte," Physical Chemistry Chemical Physics, Vol. 4, No. 12, pp. 2673-2679, 2002.

7. Yamawaki, M., Bak, T., Nowotny, M., Nowotny, J., and Sorrell, C., "Application of the High-temperature Kelvin Probe for in Situ Monitoring of the Charge Transfer at the Oxygen/Zirconia Interface. Oxygen Chemisorption Isobar," Journal of Physics and Chemistry of Solids, Vol. 66, No. 2, pp. 322-328, 2005.

8. Lee, W., Jung, H. J., Lee, M. H., Kim, Y. B., Park, J. S., et al., "Oxygen Surface Exchange at Grain Boundaries of Oxide Ion Conductors," Advanced Functional Materials, Vol. 22, No. 5, pp. 965-971, 2012.

9. Nowotny, M., Bak, T., Nowotny, J., Sorrell, C., Prince, K., et al., "Charge Transfer at Oxygen/Zirconia Interface at Elevated Temperatures: Part 9: Room Temperature," Advances in Applied Ceramics, Vol. 104, No. 4, pp. 206-213, 2005.
10. Shim, J. H., Park, J. S., Holme, T. P., Crabb, K., Lee, W., et al., "Enhanced Oxygen Exchange and Incorporation at Surface Grain Boundaries on an Oxide Ion Conductor," Acta Materialia, Vol. 60, No. 1, pp. 1-7, 2012.

11. Lee, W., Prinz, F. B., Chen, X., Nonnenmann, S., Bonnell, D. A., et al., "Nanoscale Impedance and Complex Properties in Energyrelated Systems," MRS Bulletin, Vol. 37, No. 7, pp. 659-667, 2012.

12. Lee, W., Lee, M. H., O’Hayre, R. P., and Prinz, F. B., "Nanoscale Electrochemistry in Energy Related Systems using Atomic Force Microscopy," in: Scanning Probe Microscopy for Energy Research, Materials, Devices, and Applications, Dawn, A. B., Sergei, V. K., (Eds), World Scientific Publishing Company, pp. 317-340, 2013.

13. Lee, W., Lee, M., Kim, Y. B., and Prinz, F. B., "Reduction and Oxidation of Oxide Ion conductors with Conductive Atomic Force Microscopy," Nanotechnology, Vol. 20, No. 44, Paper No. 445706, 2009.

14. Nonnenmacher, M., O’Boyle, M., and Wickramasinghe, H., "Kelvin Probe Force Microscopy,” Applied Physics Letters, Vol. 58, No. 25, pp. 2921-2923, 1991.

15. Lee, M., Lee, W., and Prinz, F. B., "Geometric Artefact Suppressed Surface Potential Measurements," Nanotechnology, Vol. 17, No. 15, Paper No. 3728, 2006.

16. Glatzel, T., Sadewasser, S., and Lux-Steiner, M. C., "Amplitude or Frequency Modulation-detection in Kelvin Probe Force Microscopy,” Applied Surface Science, Vol. 210, No. 1, pp. 84-89, 2003.

17. Huang, H., Gür, T. M., Saito, Y., and Prinz, F., "High Ionic Conductivity in Ultrathin Nanocrystalline Gadolinia-doped Ceria Films," Applied Physics Letters, Vol. 89, No. 14, Paper No. 143107, 2006.

18. Shen, Y., Lee, M., Lee, W., Barnett, D. M., Pinsky, P. M., et al., “A Resolution Study for Electrostatic Force Microscopy on Bimetallic Samples using the Boundary Element Method," Nanotechnology, Vol. 19, No. 3, Paper No. 035710, 2008.

19. Riess, I. and Vayenas, C. G., "Fermi Level and Potential Distribution in Solid Electrolyte Cells with and without Ion Spillover," Solid State Ionics, Vol. 159, No. 3, pp. 313-329, 2003.

20. Buh, G., Chung, H., and Kuk, Y., "Real-time Evolution of Trapped Charge in a SiO 2 Layer: An Electrostatic Force Microscopy Study," Applied Physics Letters, Vol. 79, No. 13, pp. 2010-2012, 2001.

21. Lambert, J., Saint-Jean, M., and Guthmann, C., "Contact Electrification of High-K Oxides Studied by Electrostatic Force Microscopy," Journal of Applied Physics, Vol. 96, No. 12, pp. 73617369, 2004.

22. Sakai, N., Yamaji, K., Horita, T., Kishimoto, H., Xiong, Y., et al., "Significant Effect of Water on Surface Reaction and Related Electrochemical Properties of Mixed Conducting Oxides," Solid State Ionics, Vol. 175, No. 1, pp. 387-391, 2004. 
23. Zhou, X. D., Huebner, W., Kosacki, I., and Anderson, H. U., "Microstructure and Grain-boundary Effect on Electrical Properties of Gadolinium-doped Ceria," Journal of the American Ceramic Society, Vol. 85, No. 7, pp. 1757-1762, 2002.

24. Goodenough, J. B., "Oxide-ion Electrolytes," Annual Review of Materials Research, Vol. 33, No. 1, pp. 91-128, 2003. 